Minireview

Esther Barreiro* and Joaquim Gea

\title{
PARP-1 and PARP-2 activity in cancer-induced cachexia: potential therapeutic implications
}

https://doi.org/10.1515/hsz-2017-0158

Received May 2, 2017; accepted October 4, 2017; previously published online October 10, 2017

Abstract: Skeletal muscle dysfunction and mass loss is a characteristic feature in patients with chronic diseases including cancer and acute conditions such as critical illness. Maintenance of an adequate muscle mass is crucial for the patients' prognosis irrespective of the underlying condition. Moreover, aging-related sarcopenia may further aggravate the muscle wasting process associated with chronic diseases and cancer. Poly(adenosine diphosphate-ribose) polymerase (PARP) activation has been demonstrated to contribute to the pathophysiology of muscle mass loss and dysfunction in animal models of cancer-induced cachexia. Genetic inhibition of PARP activity attenuated the deleterious effects seen on depleted muscles in mouse models of oncologic cachexia. In the present minireview the mechanisms whereby PARP activity inhibition may improve muscle mass and performance in models of cancer-induced cachexia are discussed. Specifically, the beneficial effects of inhibition of PARP activity on attenuation of increased oxidative stress, protein catabolism, poor muscle anabolism and mitochondrial content and epigenetic modulation of muscle phenotype are reviewed in this article. Finally, the potential therapeutic strategies of pharmacological PARP activity inhibition for the treatment of cancer-induced cachexia are also being described in this review.

Keywords: biological events; muscle mass loss; oncologic cachexia; PARP-1 and PARP-2 activity; skeletal muscles.

\footnotetext{
*Corresponding author: Dr. Esther Barreiro, Pulmonology Department, IMIM-Hospital del Mar, PRBB, Dr. Aiguader, 88, E-08003 Barcelona, Spain, e-mail: ebarreiro@imim.es Joaquim Gea: Respiratory Medicine Department, Muscle Wasting and Cachexia in Chronic Respiratory Diseases and Lung Cancer Research Group, Institute of Medical Research of Hospital del Mar (IMIM)-Hospital del Mar, Parc de Salut Mar, Barcelona Biomedical Research Park (PRBB), Barcelona, Spain; Department of Health Sciences (CEXS), Universitat Pompeu Fabra, Barcelona, Spain; and Centro de Investigación en Red de Enfermedades Respiratorias (CIBERES), Instituto de Salud Carlos III (ISCIII), Barcelona, Spain
}

\section{Introduction}

Skeletal muscle dysfunction and mass loss is a characteristic feature in patients with chronic diseases including cancer and acute conditions such as critical illness. Maintenance of an adequate muscle mass is crucial for the patients' prognosis irrespective of the underlying condition (Barreiro et al., 2015; Barreiro, 2017; Vogelmeier et al., 2017). It has been repeatedly shown that chronic patients with poor muscle mass and weakness and nutritional abnormalities die significantly earlier than those with normal body composition (Marquis et al., 2002; Swallow et al., 2007; Fearon et al., 2011; Barreiro et al., 2015; Puig-Vilanova et al., 2015). Physical activity and exercise tolerance is also diminished in patients with serious muscle mass loss and weakness, thus impairing the patients' quality of life and prognosis (Marquis et al., 2002; Swallow et al., 2007; Seymour et al., 2010; Fearon et al., 2011; Shrikrishna et al., 2012; Barreiro et al., 2015; Puig-Vilanova et al., 2015). In addition, aging-related sarcopenia may further aggravate the muscle wasting process associated with chronic diseases and cancer.

Although the underlying biology of muscle mass loss and dysfunction in chronic disorders is still under investigation, several common biological mechanisms such as oxidative stress, epigenetic alterations, inflammation, and poly(adenosine diphosphate-ribose) polymerase (PARP) activation have been demonstrated to contribute to the pathophysiology of muscle mass loss and dysfunction in animal models (Marquis et al., 2002; Swallow et al., 2007; Barreiro et al., 2011, 2015; Fearon et al., 2011; Fermoselle et al., 2012; Puig-Vilanova et al., 2014a,b,c; Barreiro and Gea, 2015; Puig-Vilanova et al., 2015; Chacon-Cabrera et al., 2015, 2017). Inhibition of PARP activity has been demonstrated to exert beneficial effects in organs and tissues including skeletal muscles and several cancer types. As several pharmacological inhibitors of PARP enzyme activity are already available, the interest in the study of PARP activity and its effects on tissues is increasingly growing. Furthermore, as current therapeutic strategies to treat muscle mass loss are still scarce, research on this arena should be welcome. In this 
regard, the present minireview aims to give an overview of the most relevant findings on the potential implications of PARP activity inhibition as a therapeutic strategy for the treatment of muscle mass loss and dysfunction in cancer-induced cachexia.

\section{PARP enzyme activity}

PARP enzymes consist of a family of proteins that catalytically cleave $\beta$-nicotinamide adenine dinucleotide $\left(\beta-\mathrm{NAD}^{+}\right)$into nicotinamide and $\mathrm{ADP}$-ribose and transfer the ADP-ribose moiety to acceptor residues of target proteins (Yelamos et al., 2011). Importantly, PARP family of nuclear proteins are involved in the regulation of several cell functions such as DNA repair, cell cycle progression, chromatin function, gene transcription, genomic stability, angiogenesis, and cell death among the most relevant ones (Hassa and Hottiger, 1999) (Figure 1). Poly(ADP-ribosyl)ation, a process characterized by the transfer of ADP-ribose moiety of $\mathrm{NAD}^{+}$molecules to acceptor residues of target proteins by PARP, is an important posttranslational modification of protein residues such as DNA replication factors and signaling molecules. Moreover, poly(ADP-ribosyl)ation may also regulate epigenetic events in cells (Chacon-Cabrera et al., 2015) (Figure 1).

\section{Pathophysiological effects of parp activity in tissues}

Among the 17 members of the PARP family, PARP-1 and PARP-2 play a fundamental role in epigenetic processes through the orchestration of several chromatin-based biological activities (Yelamos et al., 2011). As such native condensed chromatin superstructure was restored by poly(ADP-ribose) glycohydrolase in vitro (de et al., 1986). Interestingly, PARP-1 inhibition using pharmacological agents or knockout mice was also shown to increase the levels of type III histone deacetylase Sirtuin1 activity and to improve oxidative metabolism in the animals (Bai et al., 2011b). Moreover, inhibition of PARP-2 also induced an increase in mitochondrial content and Sirtuin1 protein levels in limb muscles of the knockout mice (Bai et al., 2011a). On the other hand, PARP activation may also induce deleterious effects on tissues due to the upregulation of proinflammatory cascades or by inducing a hypermetabolic state within the cells, hence leading to metabolic depletion (Ullrich et al., 1999, 2000; Bai et al., 2015).

PARP-1 binds to both single- and double-stranded DNA breaks, thus acting both as a DNA damage sensor and a signaling molecule (Figure 2). PARP-1 forms homodimers and catalyzes the cleavage of $\mathrm{NAD}^{+}$into nicotinamide and ADP-ribose upon its binding to damaged DNA.

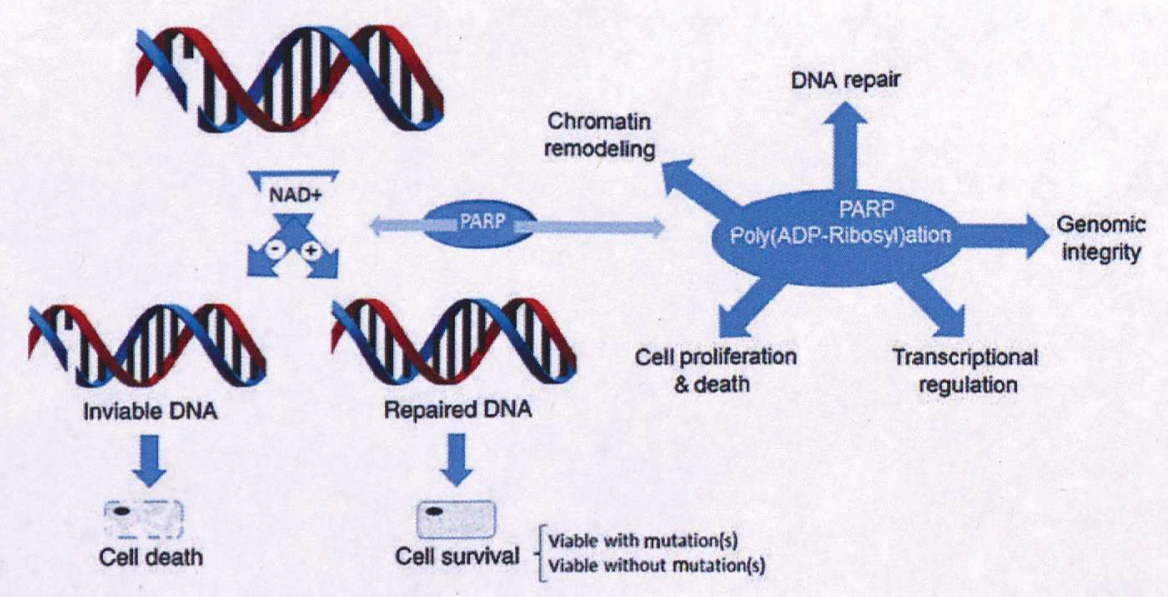

Figure 1: Schematic representation of the effects of poly (adenosine diphosphate-ribose) polymerase activity in cells.

Poly(adenosine diphosphate-ribose) polymerase (PARP) catalyzes the incorporation of ADP-ribose moiety of nicotinamide adenine dinucleotide $\left(\mathrm{NAD}^{+}\right)$into a homopolymer of repeating ADP-ribose units that covalently bind to different nuclear acceptors involved in chromatin architecture or in DNA metabolism including PARP itself. Hence, PARP family of nuclear proteins may regulate several cell functions such as DNA repair, cell cycle progression, chromatin function, gene transcription, genomic stability and cell death. At the site of DNA break, the repair process comprises primarily the catalytic transfer of the ADP-ribose moiety from NAD+ by PARP. Mutations may be transmitted in surviving cells. When severe DNA damage occurs, PARP activity may be detrimental. Thus, DNA is not repaired and cell death mechanisms are activated. 


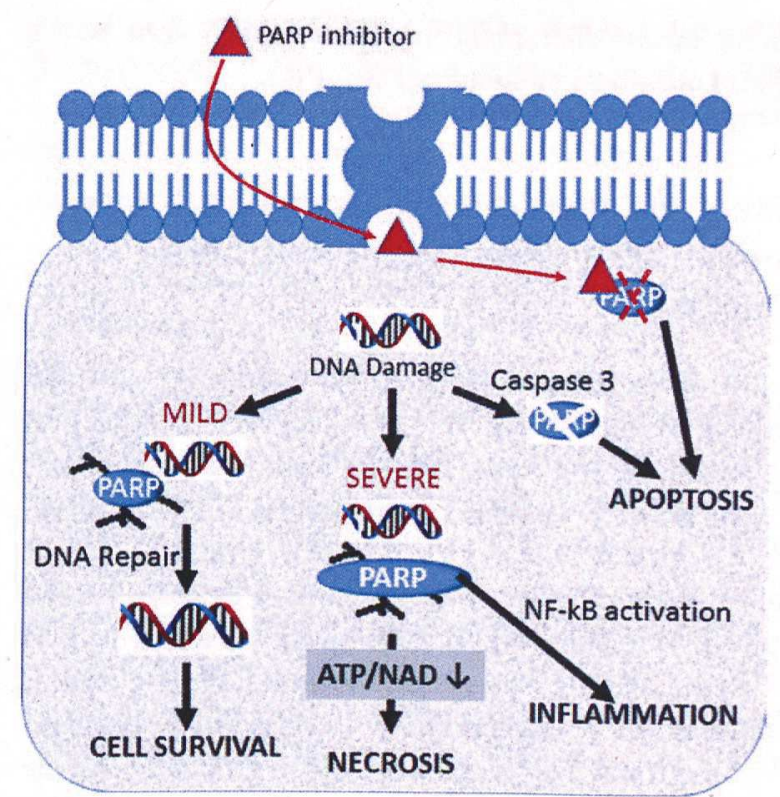

Figure 2: Poly(ADP-ribosyl)ation is characterized by the transfer of ADP-ribose moiety of $\mathrm{NAD}^{+}$molecules to acceptor residues of target proteins by PARP.

DNA damage occurs following mild damage leading to cell survival signals. However, when DNA is severely damaged signaling pathways (NF- $\mathrm{KB}$ ) are activated to induce inflammation and/or cell destruction via necrosis following depletion of ATP stores. Moreover, apoptosis may also take place following excessive DNA damage. Inhibitors of PARP activity would interfere with the actions mediated by these enzymes at different levels within cells.

Long branches of ADP-ribose polymers are formed on target proteins namely histones and PARP-1 itself (Pacher and Szabo, 2008). This process entails a severe energetic depletion, mitochondrial dysfunction, and eventual tissue destruction (necrosis) (Virag and Szabo, 2002; Schreiber et al., 2006). The signaling molecule nuclear factor (NF)$\kappa \mathrm{B}$ may also be activated by PARP-1 activity, thus leading to the activation of inflammatory and oxidative cascades of events in tissues. As such activation of PARP activity has also been shown in oxidative stress-related pathologies, and in metabolic and immune regulation (Yelamos et al., 2011; Burkle and Virag, 2013). Overactivation of PARP, which may occur as a result of increased oxidant production, exerts deleterious effects on tissues mainly by depletion of intracellular $\mathrm{NAD}^{+}$and ATP stores that lead to cell dysfunction and death (Ha and Snyder, 1999) (Figure 2). Importantly, this mechanism of cell destruction participates in the etiology of conditions characterized by severe muscle wasting, such as acute lung and renal injuries (Kiefmann et al., 2004; Zheng et al., 2005; Vaschetto et al., 2008) and sepsis (Jagtap et al., 2002). Interestingly, in cardiovascular and neurodegenerative diseases inhibition of PARP activity (genetic or pharmacological) along with neutralization of the highly reactive species peroxynitrite resulted in the prevention of cell death and attenuation of inflammatory signaling pathways (Jagtap and Szabo, 2005; Pacher et al., 2007; Szabo et al., 2007). On the other hand, PARP-1 and PARP-2 inhibition was also shown to promote oxidative metabolism and increased energy expenditure in skeletal muscles in other studies (Bai et al., 2011a,b) and to attenuate muscle mass loss in cancer cachectic mice through epigenetic regulation (Chacon-Cabrera et al., 2015).

\section{Role of PARP activity in skeletal muscles in cancer-induced cachexia}

\section{Effects on oxidative stress and proteolysis signaling}

PARP activation has been recently demonstrated in the diaphragm and gastrocnemius muscles of mice with lung cancer-induced cachexia (Chacon-Cabrera et al., 2017). Additionally, in the same investigation, levels of protein oxidation, NF- $\kappa \mathrm{B}$, tyrosine release (marker of muscle protein degradation), and ubiquitin-proteasome system markers were all increased in both respiratory and limb muscles in the mice (Chacon-Cabrera et al., 2017). The line has also been put forward that contractile myosin, mitochondrial content, and superoxide dismutase levels were reduced in the muscles of the cancer cachectic mice in the same study (Chacon-Cabrera et al., 2017). Importantly, in cancer-cachectic mice that were genetically deficient for either Parp-1 or Parp-2 expression, all these biological alterations were attenuated in their respiratory and limb muscles, while showing a significant reduction in PARP activity in those muscles (Chacon-Cabrera et al., 2017). Body weight loss was also partly attenuated in Parp$1^{1-1-}$ and Parp- $2^{-1-}$ mice bearing the lung tumor during the study period (Chacon-Cabrera et al., 2017).

Specifically, deletion of Parp-1 and Parp-2 genes attenuated the rise in protein oxidation and the decrease in SOD2 in both respiratory and limb muscles (ChaconCabrera et al., 2017). These results implied that PARP may underlie oxidant production and alter antioxidant defense in cancer cachectic muscles. Indeed, it had been demonstrated that resistance to oxidative stress was highly dependent on PARP activation (Olah et al., 2015), and that PARP also activated proteasomal degradation in response to increased oxidative stress in leukemic 
cells (Ullrich et al., 2000). In keeping with this, deletion of either Parp-1 or Parp-2 genes attenuated the decrease in myosin protein loss observed in the cancer cachectic mice (Chacon-Cabrera et al., 2017). In the study, it was also suggested that the improvements observed in body and muscle weights and limb muscle strength in Parp-1-1and Parp- $-2^{-1}$ cachectic mice was partly the result of the reduced muscle proteolysis seen in the absence of PARP activity (Chacon-Cabrera et al., 2017).

Importantly, NF- $\kappa \mathrm{B}$ and MAPK were the predominant signaling pathways driving muscle mass loss in mice with lung cancer-induced cachexia (Chacon-Cabrera et al., 2014, 2017). Increased transcriptional activity of NF- $\mathrm{KB}$ was also shown in the gastrocnemius of cancer cachectic mice (Chacon-Cabrera et al., 2014). Therefore, the decrease in proteolytic degradation observed in the respiratory and limb muscles of Parp-1-1- and Parp- $2^{-1-}$ knockout mice may be attributable to the reduced NF- $\mathrm{BB}$ transcriptional activity induced by PARP enzyme deficiency as formerly shown in different models (Hassa and Hottiger, 1999, 2008; Oliver et al., 1999).

\section{Effects on markers of muscle anabolism}

Similarly to previous reports (Fry et al., 2011; White et al., 2011; Toth et al., 2013), levels of the anabolic marker mTOR were lower in the diaphragm and gastrocnemius of cancer cachectic wild type mice (Chacon-Cabrera et al., 2017). In cachectic Parp-1-1- and Parp-2-1- rodents, the decrease in mTOR levels was attenuated in both muscles, thus suggesting that PARP-1 and PARP-2 may have interacted with mTOR signaling in cachectic muscles, at least in that experimental model (Chacon-Cabrera et al., 2017). These results were consistent with those reported in previous studies, in which pharmacological inhibitors of PARP activity were shown to prevent mTOR downregulation by modulating adenosine monophosphate-activated protein kinase pathways (Ethier et al., 2012).

Mitochondrial content as measured by the ratio of mitochondrial to nuclear DNA was reduced in diaphragm and gastrocnemius muscles of cancer cachectic mice, and such a reduction was attenuated in the cachectic mice exhibiting Parp-1 and Parp-2 genetic deletions (ChaconCabrera et al., 2017). Increased mitochondrial content was also shown upon inhibition of PARP-1, probably via Sirtuin1 activity (Rajamohan et al., 2009; Bai et al., 2015), thus suggesting that PARP activity may play a significant role in maintenance of muscle metabolism via mitochondrial content. In keeping with, inhibition of PARP-2 activity also resulted in enhanced Sirtuin1 activity and increased mitochondrial content in several in vitro models (Bai et al., 2011a; Mohamed et al., 2014).

\section{Effects on muscle-specific microRNAs and protein acetylation in cancer cachectic muscles}

As in developmental myogenesis, microRNAs and epigenetic events are also in control of muscle repair and regeneration following muscle fiber loss and injury during muscle wasting conditions such as in muscular dystrophies (Consalvi et al., 2011; Cui et al., 2017). In this respect, a potential association between PARP-1 and -2 expressions and muscle-specific microRNAs was first demonstrated in skeletal muscles of lung cancer cachectic mice (Chacon-Cabrera et al., 2015). The conclusions from that study were that particularly in the gastrocnemius of the tumor-bearing rodents, PARP-1 inhibition favored muscle proliferation and differentiation processes during regeneration as a result of the attenuation of miR-133, miR-206, and miR-486 downregulation, while PARP-2 inhibition may have rather promoted muscle differentiation of myoblasts by attenuating miR-206 downregulation. The conclusions from the study were that the blockade of PARP activity may have interacted with NF- $\kappa \mathrm{B}$, which has been consistently demonstrated to be upregulated in muscle wasting processes (Fermoselle et al., 2012, 2013; Puig-Vilanova et al., 2014c; Chacon-Cabrera et al., 2015). In fact, PARP-1 had been previously shown to directly interact with nuclear factor (NF)- $\mathrm{kB}$ (Hassa and Hottiger, 1999, 2008). In this regard, inhibition of PARP activity, especially of PARP-1, may have prevented a further decrease in the expression of muscle-specific microRNAs by blocking NF- $\mathrm{\kappa B}$ activity in vivo, particularly in the limb muscle of the cancer cachectic mice (Chacon-Cabrera et al., 2015).

Hyperacetylation of proteins, which relies to a great extent on histone deacetylase (HDAC) activity, may lead to muscle mass loss in vivo by rendering proteins more prone to degradation through the action of histone acetyl transferases that may have ubiquitin-ligase activity and by dissociation of proteins from cellular chaperones (Alamdari et al., 2013). Total protein acetylation levels were increased in the respiratory and limb muscles of lung cancer cachectic mice, while those of HDAC3, HDAC6, and Sirtuin1 were decreased (Chacon-Cabrera et al., 2015). Similar findings had also been reported in in vivo models of muscle mass loss in previous studies (Sadoul et al., 2008; Alamdari et al., 2010, 2013; Puig-Vilanova et al., 2015), thus implying that reduced HDAC activity drives protein hyperacetylation 
in skeletal muscles during muscle wasting conditions (Sadoul et al., 2008; Alamdari et al., 2010, 2013; PuigVilanova et al., 2015). A relevant finding in the study was that increased total protein acetylation levels and reduced HDAC and Sirtuin 1 content were attenuated in the muscles of Parp-1-/ and Parp-2-/- cachectic mice, which suggested that PARP-1 and PARP-2 inhibition may have prevented the cachectic muscles from undergoing further protein acetylation (Chacon-Cabrera et al., 2015).

Levels of acetylation of specific transcription factors involved in muscle metabolism and mass maintenance have also been quantified in cancer cachectic muscles of mice (Chacon-Cabrera et al., 2015). As such in wild type lung cancer cachectic mice, acetylation levels of FoxO3 were significantly increased in the diaphragm and gastrocnemius, while a rise in acetylated FoxO1 levels was only seen in the respiratory muscle of the same animals (Chacon-Cabrera et al., 2015). Importantly, no significant differences were seen in either FoxO1 or FoxO3 acetylation levels in the respiratory or limb muscles in Parp-1-1- and Parp-2- cancer cachectic mice. As previously demonstrated in vitro (Tseng et al., 2014), these findings imply that acetylation of the atrophy signaling pathways favored muscle protein loss, which were partly prevented by PARP-1 and PARP-2 activity inhibition in the cachectic mice (Chacon-Cabrera et al., 2015).

Myogenic regulatory factors control myogenesis and muscle remodeling in response to injury in adult muscles. Furthermore, the fiber type profile of a given muscle may also be orchestrated by the myogenic regulatory factors. As such, MEF2 family of transcription factors plays a relevant role in muscle phenotype determination of fast- and slow-twitch muscle fibers in mice (Potthoff et al., 2007). Moreover, MEF2 is regulated by acetylation and deacetylation events such as class II HDACs, which can directly bind and inhibit MEF2-regulated transcription of genes (Zhang et al., 2002). In muscles of lung cancer cachectic mice, protein levels of MEF2C and MEF2D were reduced, whereas such a reduction was attenuated in the same muscles of Parp- $-^{-1-}$ and Parp-2-1- cachectic mice (ChaconCabrera et al., 2015). Yin Yang (YY)1 is a transcription factor involved in histone modifications, which inhibits muscle regeneration through the transcriptional silencing of myofibrillar genes (Wang et al., 2007). In a previous investigation (Natanek et al., 2011), protein levels of YY1 inversely correlated with the reduced size of slow- and fast-twitch muscle fibers in the vastus lateralis of patients with COPD. Recently, YY1 levels were shown to be reduced in respiratory and limb muscles of cancer cachectic mice including those exhibiting Parp-1 or Parp-2 genetic deletions (Chacon-Cabrera et al., 2015). The conclusions from

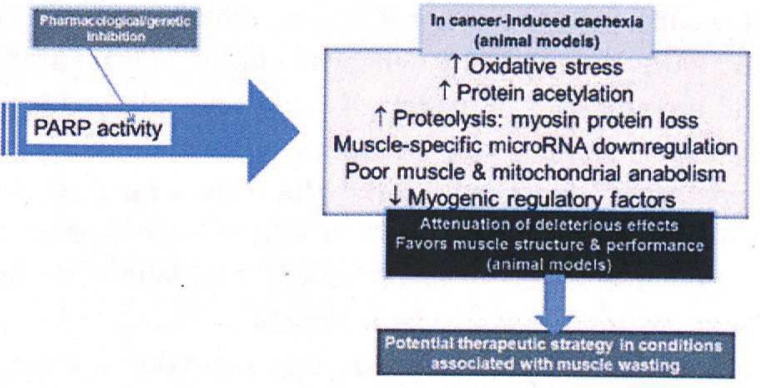

Figure 3: Brief outline of the biological mechanisms through which genetic inhibition of PARP activity improves muscle mass loss and dysfunction in models of cancer-induced cachexia.

Biological events such as increased oxidative stress, protein acetylation, and proteolysis together with reduced muscle-specific microRNA expression and myogenic regulatory factors, and poor muscle mass mitochondrial anabolism have been demonstrated in the skeletal muscles of mice with cancer cachexia. Inhibition of PARP activity partially reversed these deleterious events in the mouse muscles, leading to improvements in muscle structure and performance. These results led to the concept that inhibition of PARP activity may be a good therapeutic approach in cancer cachexia. For a review see Chacon-Cabrera et al. $(2015,2017)$.

those findings were that as the deficiency of PARP- 1 and PARP-2 activity induced an increase in the size of slow- and fast-twitch fibers in both respiratory and limb muscles of the cancer cachectic animals, YY1 most likely contributed to the maintenance of fiber size seen in those muscles (Chacon-Cabrera et al., 2015) (Figure 3).

\section{Concluding remarks and future perspectives}

The potential beneficial effects of parp-1 and parp-2 genetic deletions to prevent cancer-induced cachexia have been explored on the basis of different investigations based on the use of specific in vivo mouse models. The results gathered so far have demonstrated that the absence of PARP activity mitigated the expression of several biological events that are involved in the process of poor muscle anabolism, mass loss and weakness in cancer-induced cachexia. Moreover, the attenuation of the biological events was accompanied by the improvement in muscle structural features, especially atrophy, and performance in the cancer cachectic mice along with an amelioration of the rates of their body weight loss.

As pharmacological inhibitors of PARP-1 and - 2 are currently available for the treatment of certain cancer types in humans, future research should focus on the assessment 
of whether selective pharmacological inhibitors of PARP-1 and PARP-2 exert similar beneficial effects on respiratory and limb muscles in models of cancer-induced cachexia including patients with cancer and muscle wasting. Nonetheless, the potential harmful effects on genomic instability derived from PARP activity inhibition should be primarily identified before PARP-1/2 inhibitors can be routinely prescribed for the treatment of cancer-induced cachexia or other muscle wasting conditions. Future avenues of research should be designed to specifically address this question. Those findings would facilitate the design of selective PARP inhibitors that may promote the restoration of both muscle mass and strength in patients with oncologic cachexia. Clinical trials to specifically test whether pharmacological PARP inhibitors may improve muscle mass and function in patients with chronic debilitating conditions that are associated with muscle wasting are urgently needed. Such improvements would have beneficial effects on the patients' exercise capacity, quality of life, and disease prognosis.

Acknowledgments: Editorial support: None to declare. CIBERES, FIS 14/00713 (FEDER), SAF-2014-54371-R, SEPAR 2016, and FUCAP 2016 (Spain) have contributed to support part of the research described in this review.

Conflict of interest statement: The authors declare no conflict of interest, financial or otherwise in relation to this article.

\section{References}

Alamdari, N., Aversa, Z., Castillero, E., and Hasselgren, P.O. (2013) Acetylation and deacetylation - novel factors in muscle wasting. Metabolism 62,1-11.

Alamdari, N., Smith, I.J., Aversa, Z., and Hasselgren, P.O. (2010) Sepsis and glucocorticoids upregulate p300 and downregulate HDAC 6 expression and activity in skeletal muscle. Am. J. Physiol. Regul. Integr. Comp. Physiol. 299, R509-R520.

Bai, P., Canto, C., Brunyanszki, A., Huber, A., Szanto, M., Cen, Y., Yamamoto, H., Houten, S.M., Kiss, B., Oudart, H., et al. (2011a) PARP-2 regulates SIRT1 expression and whole-body energy expenditure. Cell Metab. 13, 450-460.

Bai, P., Canto, C., Oudart, H., Brunyanszki, A., Cen, Y., Thomas, C., Yamamoto, H., Huber, A., Kiss, B., Houtkooper, R.H., et al. (2011b) PARP-1 inhibition increases mitochondrial metabolism through SIRT1 activation. Cell Metab. 13, 461-468.

Bai, P., Nagy, L., Fodor, T., Liaudet, L., and Pacher, P. (2015) Poly(ADP-ribose) polymerases as modulators of mitochondrial activity. Trends Endocrinol. Metab. 26, 75-83.

Barreiro, E. (2017) Skeletal muscle dysfunction in COPD: novelties in the last decade. Arch. Bronconeumol. 53, 43-44.
Barreiro, E. and Gea, J. (2015) Epigenetics and muscle dysfunction in chronic obstructive pulmonary disease. Transl. Res.165, 61-73.

Barreiro, E., Ferrer, D., Sanchez, F., Minguella, J., Marin-Corral, J., Martinez-Llorens, J., Lloreta, J., and Gea, J. (2011) Inflammatory cells and apoptosis in respiratory and limb muscles of patients with COPD. J. Appl. Physiol. 111, 808-817.

Barreiro, E., Bustamante, V., Cejudo, P., Galdiz, J.B., Gea, J., de, L.P., Martinez-Llorens, J., Ortega, F., Puente-Maestu, L., Roca, J., et al. (2015) Guidelines for the evaluation and treatment of muscle dysfunction in patients with chronic obstructive pulmonary disease. Arch. Bronconeumol. 51, 384-395.

Burkle, A. and Virag, L. (2013) Poly(ADP-ribose): PARadigms and PARadoxes. Mol. Aspects Med. 34, 1046-1065.

Chacon-Cabrera, A., Fermoselle, C., Urtreger, A.J., Mateu-Jimenez, M., Diament, M.J., De Kier Joffe, E.D., Sandri, M., and Barreiro, E. (2014) Pharmacological strategies in lung cancer-induced cachexia: effects on muscle proteolysis, autophagy, structure, and weakness. J. Cell Physiol. 229, 1660-1672.

Chacon-Cabrera, A., Fermoselle, C., Salmela, I., Yelamos, J., and Barreiro, E. (2015) MicroRNA expression and protein acetylation pattern in respiratory and limb muscles of Parp-1-1- and Parp$2^{-1-}$ mice with lung cancer cachexia. Biochim. Biophys. Acta 1850, 2530-2543.

Chacon-Cabrera, A., Mateu-Jimenez, M., Langohr, K., Fermoselle, C., Garcia-Arumi, E., Andreu, A.L., Yelamos, J., and Barreiro, E. (2017) Role of PARP activity in lung cancer-induced cachexia: effects on muscle oxidative stress, proteolysis, anabolic markers, and phenotype. J. Cell Physiol. 232, 3744-3761.

Consalvi, S., Saccone, V., Giordani, L., Minetti, G., Mozzetta, C., and Puri, P.L. (2011) Histone deacetylase inhibitors in the treatment of muscular dystrophies: epigenetic drugs for genetic diseases. Mol. Med. 17, 457-465.

Cui, H., Bansal, V., Grunert, M., Malecova, B., Dall'Agnese, A., Latella, L., Gatto, S., Ryan, T., Schulz, K., Chen, W., et al. (2017) Muscle-relevant genes marked by stable $\mathrm{H} 3 \mathrm{~K} 4 \mathrm{me} 2 / 3$ profiles and enriched MyoD binding during myogenic differentiation. PLoS One 12, e0179464.

de, M.G., Huletsky, A., Lamarre, D., Gaudreau, A., Pouyet, J., Daune, M., and Poirier, G.G. (1986) Modulation of chromatin superstructure induced by poly(ADP-ribose) synthesis and degradation. J. Biol. Chem. 261, 7011-7017.

Ethier, C., Tardif, M., Arul, L., and Poirier, G.G. (2012) PARP-1 modulation of mTOR signaling in response to a DNA alkylating agent. PLoS One 7, e47978.

Fearon, K., Strasser, F., Anker, S.D., Bosaeus, I., Bruera, E., Fainsinger, R.L., Jatoi, A., Loprinzi, C., MacDonald, N., Mantovani, G., et al. (2011) Definition and classification of cancer cachexia: an international consensus. Lancet Oncol. 12, 489-495.

Fermoselle, C., Rabinovich, R., Ausin, P., Puig-Vilanova, E., Coronell, C., Sanchez, F., Roca, J., Gea, J., and Barreiro, E. (2012) Does oxidative stress modulate limb muscle atrophy in severe COPD patients? Eur. Respir. J. 40, 851-862.

Fermoselle, C., Garcia-Arumi, E., Puig-Vilanova, E., Andreu, A.L., Urtreger, A.J., De Kier Joffe, E.D., Tejedor, A., Puente-Maestu, L., and Barreiro, E. (2013) Mitochondrial dysfunction and therapeutic approaches in respiratory and limb muscles of cancer cachectic mice. Exp. Physiol. 98, 1349-1365.

Fry, C.S., Drummond, M.J., Glynn, E.L., Dickinson, J.M., Gundermann, D.M., Timmerman, K.L., Walker, D.K., Dhanani, S., Volpi, E., 
and Rasmussen, B.B. (2011) Aging impairs contraction-induced human skeletal muscle mTORC1 signaling and protein synthesis. Skeletal Muscle 1, 1-11.

Ha, H.C. and Snyder, S.H. (1999) Poly(ADP-ribose) polymerase is a mediator of necrotic cell death by ATP depletion. Proc. Natl. Acad. Sci. USA 96, 13978-13982.

Hassa, P.O. and Hottiger, M.O. (1999) A role of poly (ADP-ribose) polymerase in $\mathrm{NF}-\mathrm{KB}$ transcriptional activation. Biol. Chem. 380, 953-959.

Hassa, P.O. and Hottiger, M.O. (2008) The diverse biological roles of mammalian PARPS, a small but powerful family of poly-ADPribose polymerases. Front Biosci. 13, 3046-3082.

Jagtap, P., Soriano, F.G., Virag, L., Liaudet, L., Mabley, J., Szabo, E., Hasko, G., Marton, A., Lorigados, C.B., Gallyas, F., Jr., et al. (2002) Novel phenanthridinone inhibitors of poly (adenosine $5^{\prime}$-diphosphate-ribose) synthetase: potent cytoprotective and antishock agents. Crit. Care Med. 30, 1071-1082.

Jagtap, P. and Szabo, C. (2005) Poly(ADP-ribose) polymerase and the therapeutic effects of its inhibitors. Nat. Rev. Drug Discov. 4, 421-440.

Kiefmann, R., Heckel, K., Doerger, M., Schenkat, S., Kupatt, C., Stoeckelhuber, M., Wesierska-Gadek, J., and Goetz, A.E. (2004) Role of PARP on iNOS pathway during endotoxin-induced acute lung injury. Intensive Care Med. 30, 1421-1431.

Marquis, K., Debigare, R., Lacasse, Y., LeBlanc, P., Jobin, J., Carrier, G., and Maltais, F. (2002) Midthigh muscle cross-sectional area is a better predictor of mortality than body mass index in patients with chronic obstructive pulmonary disease. Am. J. Respir. Crit Care Med. 166, 809-813.

Mohamed, J.S., Hajira, A., Pardo, P.S., and Boriek, A.M. (2014) MicroRNA-149 inhibits PARP-2 and promotes mitochondrial biogenesis via SIRT-1/PGC-1alpha network in skeletal muscle. Diabetes 63, 1546-1559.

Natanek, S.A., Riddoch-Contreras, J., Marsh, G.S., Hopkinson, N.S., Man, W.D., Moxham, J., Polkey, M.I., and Kemp, P.R. (2011) Yin Yang 1 expression and localisation in quadriceps muscle in COPD. Arch. Bronconeumol. 47, 296-302.

Olah, G., Szczesny, B., Brunyanszki, A., Lopez-Garcia, I.A., Gero, D., Radak, Z., and Szabo, C. (2015) Differentiation-associated downregulation of poly(ADP-Ribose) polymerase-1 expression in myoblasts serves to increase their resistance to oxidative stress. PLoS One 10, e0134227.

Oliver, F.J., Menissier-de, M.J., Nacci, C., Decker, P., Andriantsitohaina, R., Muller, S., de la Rubia, G., Stoclet, J.C., and de, M.G. (1999) Resistance to endotoxic shock as a consequence of defective NF-KB activation in poly (ADP-ribose) polymerase-1 deficient mice. EMBO J. 18, 4446-4454.

Pacher, P., Beckman, J.S., and Liaudet, L. (2007) Nitric oxide and peroxynitrite in health and disease. Physiol Rev. 87, 315-424.

Pacher, P. and Szabo, C. (2008) Role of the peroxynitrite-poly(ADPribose) polymerase pathway in human disease. Am.J.Pathol. 173, 2-13.

Potthoff, M.J., Wu, H., Arnold, M.A., Shelton, J.M., Backs, J., McAnally, J., Richardson, J.A., Bassel-Duby, R., and Olson, E.N. (2007) Histone deacetylase degradation and MEF2 activation promote the formation of slow-twitch myofibers. J. Clin. Invest. 117, 2459-2467.

Puig-Vilanova, E., Aguilo, R., Rodriguez-Fuster, A., Martinez-Llorens, J., Gea, J., and Barreiro, E. (2014a) Epigenetic mechanisms in respiratory muscle dysfunction of patients with chronic obstructive pulmonary disease. PLoS One 9, e111514.
Puig-Vilanova, E., Ausin, P., Martinez-Llorens, J., Gea, J., and Barreiro, E. (2014b) Do epigenetic events take place in the vastus lateralis of patients with mild chronic obstructive pulmonary disease? PLoS One 9, e102296.

Puig-Vilanova, E., Rodriguez, D.A., Lloreta, J., Ausin, P., Pascual-Guardia, S., Broquetas, J., Roca, J., Gea, J., and Barreiro, E. (2014c) Oxidative stress, redox signaling pathways, and autophagy in cachectic muscles of male patients with advanced COPD and lung cancer. Free Radic. Biol. Med. 79C, 91-108.

Puig-Vilanova, E., Martinez-Llorens, J., Ausin, P., Roca, J., Gea, J., and Barreiro, E. (2015) Quadriceps muscle weakness and atrophy are associated with a differential epigenetic profile in advanced COPD. Clin. Sci. (Lond) 128, 905-921.

Rajamohan, S.B., Pillai, V.B., Gupta, M., Sundaresan, N.R., Birukov, K.G., Samant, S., Hottiger, M.O., and Gupta, M.P. (2009) SIRT1 promotes cell survival under stress by deacetylation-dependent deactivation of poly(ADP-ribose) polymerase 1. Mol. Cell Biol. 29, 4116-4129.

Sadoul, K., Boyault, C., Pabion, M., and Khochbin, S. (2008) Regulation of protein turnover by acetyltransferases and deacetylases. Biochimie 90, 306-312.

Schreiber, V., Dantzer, F., Ame, J.C., and de, M.G. (2006) Poly(ADPribose): novel functions for an old molecule. Nat. Rev. Mol. Cell Biol. 7, 517-528.

Seymour, J.M., Spruit, M.A., Hopkinson, N.S., Natanek, S.A., Man, W.D., Jackson, A., Gosker, H.R., Schols, A.M., Moxham, J., Polkey, M.I., et al. (2010) The prevalence of quadriceps weakness in COPD and the relationship with disease severity. Eur. Respir. J. 36, 81-88.

Shrikrishna, D., Patel, M., Tanner, R.J., Seymour, J.M., Connolly, B.A., Puthucheary, Z.A., Walsh, S.L., Bloch, S.A., Sidhu, P.S., Hart, N., et al. (2012) Quadriceps wasting and physical inactivity in patients with COPD. Eur. Respir. J. 40, 1115-1122.

Swallow, E.B., Reyes, D., Hopkinson, N.S., Man, W.D., Porcher, R., Cetti, E.J., Moore, A.J., Moxham, J., and Polkey, M.I. (2007) Quadriceps strength predicts mortality in patients with moderate to severe chronic obstructive pulmonary disease. Thorax 62, 115-120.

Szabo, C., Ischiropoulos, H., and Radi, R. (2007) Peroxynitrite: biochemistry, pathophysiology and development of therapeutics. Nat. Rev. Drug Discov. 6, 662-680.

Toth, M.J., Miller, M.S., Callahan, D.M., Sweeny, A.P., Nunez, I., Grunberg, S.M., Der-Torossian, H., Couch, M.E., and Dittus, K. (2013) Molecular mechanisms underlying skeletal muscle weakness in human cancer: reduced myosin-actin cross-bridge formation and kinetics. J. Appl. Physiol 114 , 858-868.

Tseng, A.H., Wu, L.H., Shieh, S.S., and Wang, D.L. (2014) SIRT3 interactions with $\mathrm{FOXO} 3$ acetylation, phosphorylation and ubiquitinylation mediate endothelial cell responses to hypoxia. Biochem. J. 464, 157-168.

Ullrich, O., Reinheckel, T., Sitte, N., Hass, R., Grune, T., and Davies, K.J. (1999) Poly-ADP ribose polymerase activates nuclear proteasome to degrade oxidatively damaged histones. Proc. Natl. Acad. Sci. USA 96, 6223-6228.

Ullrich, O., Ciftci, O., and Hass, R. (2000) Proteasome activation by poly-ADP-ribose-polymerase in human myelomonocytic cells after oxidative stress. Free Radic. Biol. Med. 29 , 995-1004. 
Vaschetto, R., Kuiper, J.W., Chiang, S.R., Haitsma, J.J., Juco, J.W., Uhlig, S., Plotz, F.B., Della, C.F., Zhang, H., and Slutsky, A.S. (2008) Inhibition of poly(adenosine diphosphate-ribose) polymerase attenuates ventilator-induced lung injury. Anesthesiology 108, 261-268.

Virag, L. and Szabo, C. (2002) The therapeutic potential of poly(ADP-ribose) polymerase inhibitors. Pharmacol. Rev. 54, 375-429.

Vogelmeier, C.F., Criner, G.J., Martinez, F.J., Anzueto, A., Barnes, P.J., Bourbeau, J., Celli, B.R., Chen, R., Decramer, M., Fabbri, L.M., et al. (2017) Global strategy for the diagnosis, management, and prevention of chronic obstructive lung disease 2017 report: gold executive summary. Arch. Bronconeumol. 53, 128-149.

Wang, H., Hertlein, E., Bakkar, N., Sun, H., Acharyya, S., Wang, J., Carathers, M., Davuluri, R., and Guttridge, D.C. (2007) NF- $\kappa B$ regulation of $\mathrm{YY} 1$ inhibits skeletal myogenesis through tran- scriptional silencing of myofibrillar genes. Mol. Cell Biol: 27 4374-4387.

White, J.P., Baynes, J.W., Welle, S.L., Kostek, M.C., Matesic, L.E., Sato, S., and Carson, J.A. (2011) The regulation of skeletal muscle protein turnover during the progression of cancer cachexia in the Apc(Min/+) mouse. PLoS One 6, e24650.

Yelamos, J., Farres, J., Llacuna, L., Ampurdanes, C., and MartinCaballero, J. (2011) PARP-1 and PARP-2: new players in tumour development. Am. J. Cancer Res. 1, 328-346.

Zhang, C.L., McKinsey, T.A., and Olson, E.N. (2002) Association of class II histone deacetylases with heterochromatin protein 1: potential role for histone methylation in control of muscle differentiation. Mol. Cell Biol. 22, 7302-7312.

Zheng, J., Devalaraja-Narashimha, K., Singaravelu, K., and Padanilam, B.J. (2005) Poly(ADP-ribose) polymerase-1 gene ablation protects mice from ischemic renal injury. Am. J. Physiol. Renal Physiol. 288, F387-F398. 